\title{
Quantum Computing with Spatially Delocalized Qubits
}

\author{
J. Mompart, ${ }^{1,2}$ K. Eckert, ${ }^{1}$ W. Ertmer, ${ }^{3}$ G. Birk1,${ }^{3}$ and M. Lewenstein ${ }^{1}$ \\ ${ }^{1}$ Institute of Theoretical Physics, University of Hannover, Appelstrasse 2, D-30167 Hannover, Germany \\ ${ }^{2}$ Departament de Física, Universitat Autònoma de Barcelona, E-08193 Bellaterra, Spain \\ ${ }^{3}$ Institute of Quantum Optics, University of Hannover, Welfengarten 1, D-30167 Hannover, Germany
}

(Received 1 October 2002; published 8 April 2003)

\begin{abstract}
We analyze the operation of quantum gates for neutral atoms with qubits that are delocalized in space, i.e., the computational basis states are defined by the presence of a neutral atom in the ground state of one out of two trapping potentials. The implementation of single-qubit gates as well as a controlled phase gate between two qubits is discussed and explicit calculations are presented for rubidium atoms in optical microtraps. Furthermore, we show how multiqubit highly entangled states can be created in this scheme.
\end{abstract}

DOI: 10.1103/PhysRevLett.90.147901

PACS numbers: 03.67.Lx, 32.80.Pj, 42.50.-p

Optical lattices [1] and arrays of optical microtraps [2] are promising candidates for the implementation of quantum information processing with neutral atoms. Many of the requirements for quantum computation with optical microtraps have been recently demonstrated in $2 \mathrm{D}$ arrays of $\sim 80$ traps with $\sim 100$ atoms per trap [2]. Some remarkable characteristics of optical microtraps are the possibility to scale, miniaturize, and parallelize the required atom optics devices. In addition, they offer two fundamental advantages over optical lattices: (i) the possibility of individually addressing single traps due to the large separation of the microlenses foci, e.g., $\sim 125 \mu \mathrm{m}$; and (ii) the independent displacement of rows and columns of microtraps and, eventually, of single microtraps. Single atoms in dipole traps [3] and the Mott insulator transition with one atom per trap in optical lattices [4] have been reported, and, therefore, the achievement of $1 \mathrm{D}$ and $2 \mathrm{D}$ arrays of optical microtraps containing none or one atom per trap in a deterministic way can be foreseen for the near future. We will make use of all these features of optical microtraps to propose a novel implementation for quantum information processing.

In our scheme, each qubit consists of two traps separated by a distance $2 a$ and one single atom. Per definition, the detection of the atom in the ground state of the left trap represents $|0\rangle$ and in the right trap $|1\rangle$, i.e., $|0\rangle=|0\rangle_{L}$ and $|1\rangle=|0\rangle_{R}$, where $|0\rangle_{L, R}$ are the vibrational ground states of the left and right trap, respectively. Throughout this Letter we will call this implementation the spatially delocalized qubit (SDQ), since $|\langle 0|\vec{r}| 0\rangle-\langle 1|\vec{r}| 1\rangle|=2 a$ with $\vec{r}$ the position operator. To implement the SDQ we will assume that we are able to deterministically store none or one single atom per trap and cool it to the vibrational ground state in 3D.

Single and two-qubit gate operations will be performed by adiabatically approaching two traps which will be modeled as follows: The initial separation of the traps is $2 a_{\max }$ and to approach them to the minimum separation $2 a_{\min }$ takes a raising time $t_{r}$. The temporal evolution of the distance $a$ is described by the first half of a period of a cosine. The two wells remain at the minimum separation for an interaction time $t_{i}$ and, finally, are adiabatically separated to the initial distance. To simplify the numerical analysis we will assume piecewise harmonic trapping potentials as in Ref. [5] and, eventually, consider realistic Gaussian potentials as they are present in the experiment $[2,6]$.

Single-qubit operations, e.g., a Hadamard gate, are performed by adiabatically approaching the traps and allowing tunneling to take place. In order to illustrate this operation, it is convenient to consider the two lowest energy eigenstates of the double well potential. These two states are symmetric and antisymmetric, denoted by $|S\rangle$ and $|A\rangle$, respectively, with energies $E_{S, A}(a)=$ $E(a) \mp \frac{1}{2} \hbar \Omega(a)$ and $\Omega$ being the splitting frequency. In terms of these states, our qubit basis reads: $|0\rangle=\frac{1}{\sqrt{2}}(|S\rangle+$ $|A\rangle)$, and $|1\rangle=\frac{1}{\sqrt{2}}(|S\rangle-|A\rangle)$. Let us assume that the atom is initially in the left trap, i.e, $|\psi(t=0)\rangle=|0\rangle$, then it is straightforward to check that its time evolution will be given by $|\psi(t)\rangle=e^{-i(E t / \hbar)}[\cos (\Omega t / 2)|0\rangle+i \sin (\Omega t / 2)|1\rangle]$. Thus, the atomic wave function oscillates in a Rabi-type fashion between left and right traps at the flopping frequency $\frac{1}{2} \Omega(a)$. Obviously, for large trap separations states $|S\rangle$ and $|A\rangle$ become degenerate in energy, i.e., $\Omega(a)=0$ for $a \rightarrow \infty$, and then the atom does not evolve in time (up to a trivial phase). Therefore, it is possible to realize singlequbit operations via tunneling by experimentally controlling the "Rabi frequency" through $a_{\min }, t_{r}$, and $t_{i}$.

These single-qubit operations are illustrated in Fig. 1 through a numerical integration of the 1D Schrödinger equation in the parameter plane $t_{r}$ versus $t_{i}$ with $|0\rangle$ being the initial state. Figure 1 shows (a) the population of state $|1\rangle$, denoted by $\rho_{1}$, and (b) the total ground state population of left and right traps, i.e., $\rho_{0}+\rho_{1}$, after the whole cycle of approaching and separating the traps. This oscillating population resembles the Rabi flopping in the interaction of a laser with a two-level system and, for this reason, we have added in Fig. 1 dashed lines indicating 


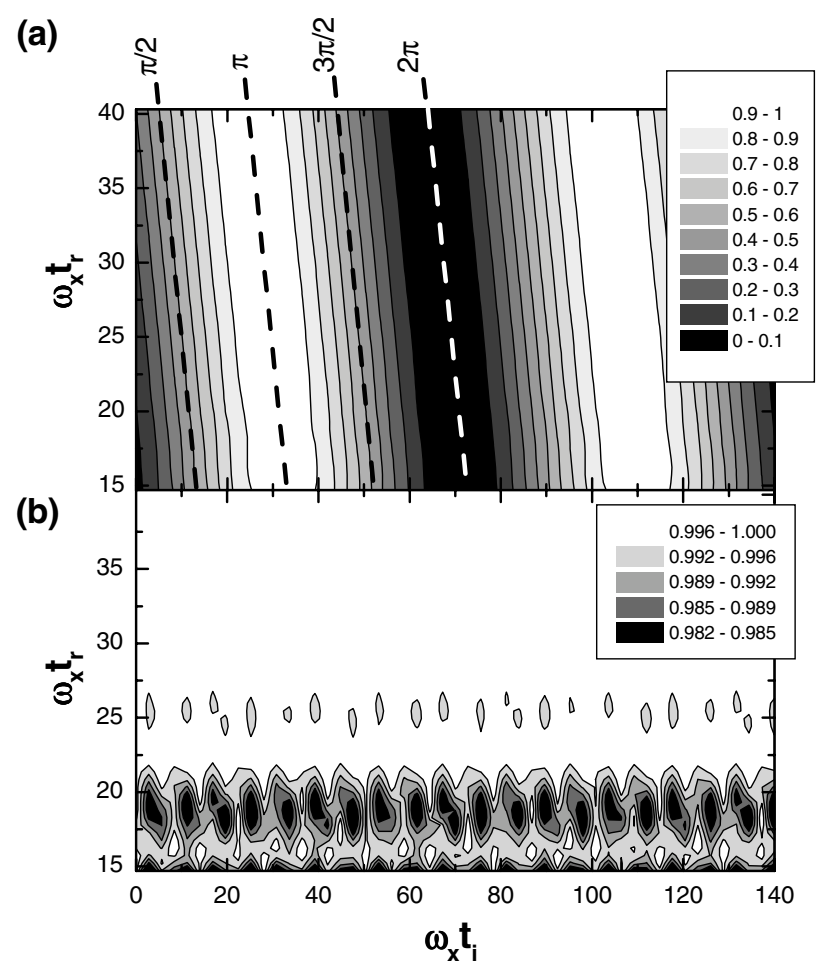

FIG. 1. Single qubit operations for $\alpha a_{\max }=5, \alpha a_{\min }=1.8$, and the atom initially in the left trap: (a) Population of the right trap; (b) the sum of the ground state population of left plus right traps. $\alpha^{-1}=\sqrt{\hbar / m \omega_{x}}$ is the position spread of the ground state with $m$ the mass of the neutral atom and $\omega_{x}$ the trapping frequency.

the $\frac{\pi}{2}, \pi, \frac{3 \pi}{2}, 2 \pi$ laser pulse notation conventionally used in quantum optics. For small $t_{r}$, nonadiabaticity results in the population of excited vibrational states, which, as shown in Fig. 1(b), yields $\rho_{0}+\rho_{1}<1$. In what follows, we will focus only in the adiabatic regime.

For the two-qubit gate operations we assume that the two qubits are arranged either (i) in a 1D configuration, i.e., the four traps form a line, or (ii) side-by-side in a $2 \mathrm{D}$ configuration, i.e., the traps form a square, cf. Fig. 2(a). The traps involved are labeled $A_{0}, A_{1}$ for the first qubit and $B_{0}, B_{1}$ for the second, and the respective ground states are denoted $|0\rangle_{A},|1\rangle_{A}$ and $|0\rangle_{B},|1\rangle_{B}$. A two-qubit gate will be realized via the collisional interaction between bosonic neutral atoms [7]. We will consider ${ }^{87} \mathrm{Rb}$ atoms whose collisional interaction can be described by a contact potential of the form $U\left(\vec{r}_{1}, \vec{r}_{2}\right)=$ $4 \pi a_{t} \hbar^{2} m^{-1} \delta^{3}\left(\vec{r}_{1}-\vec{r}_{2}\right)$, where $a_{t}=106 a_{0}=5.61 \mathrm{~nm}$ is the $s$-wave scattering length in the spin triplet. Because the states $|0\rangle$ and $|1\rangle$ are localized in different positions, it is enough to perform a suitable spatiotemporal variation of the potentials in order to pick up a collisional phase shift, e.g., only if both atoms are in $|1\rangle$. This is sufficient to implement a two-qubit phase gate, which transforms product states $|i\rangle_{A}|j\rangle_{B}, \quad i, j \in\{0,1\}$, into $\exp \left(i \delta_{i 1} \delta_{j 1} \pi\right)|i\rangle_{A}|j\rangle_{B}$ and, supplemented by arbitrary single-qubit gates, forms a universal set of gates.
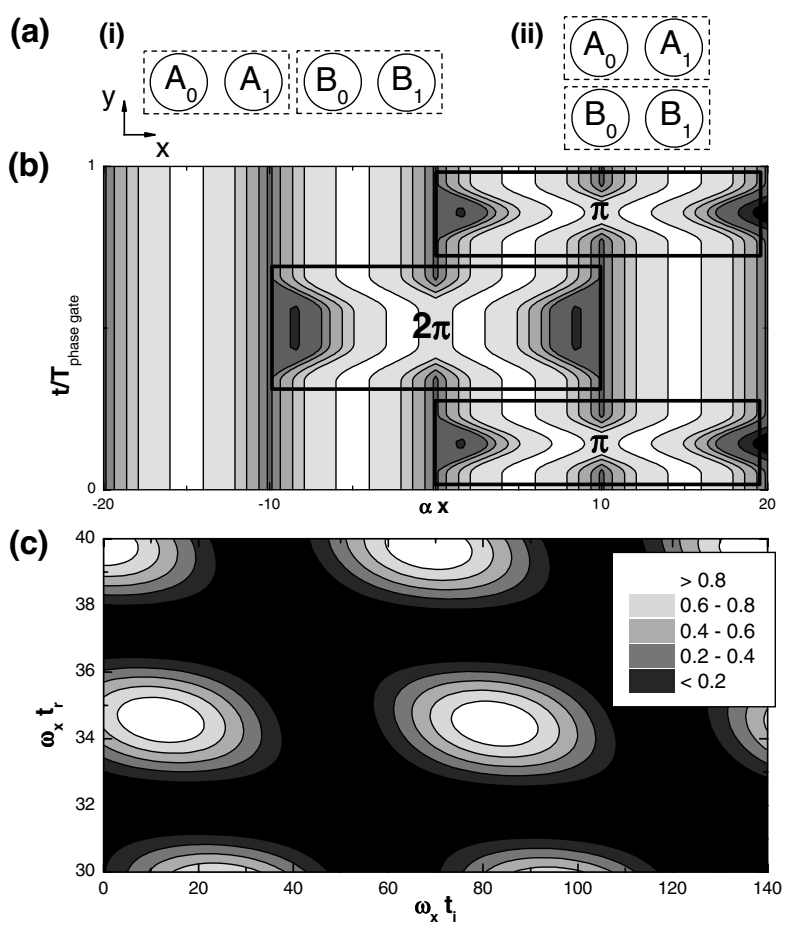

FIG. 2. Two-qubit phase gate operation. (a) Arrangements of the qubits: (i) in-line, and (ii) side-by-side; (b) Contourplot of the spatiotemporal variation of the trapping potential for arrangement (i). The centers of the four traps are white; dark gray means high potential energy; (c) Fidelity $F=$ $\rho\left[\cos \left(\phi_{C}-\pi\right)+1\right] / 2$. Parameters as in Fig. 1 with $a_{t}=$ $106 a_{0}, \omega_{x}=\omega_{y}=2 \times 10^{5} \mathrm{~s}^{-1}$, and $\omega_{z}=1.1 \times 10^{6} \mathrm{~s}^{-1}$.

For the inline arrangement, the change of the potential leading to a phase gate is shown in Fig. 2(b), where horizontal and vertical axes denote space and time, respectively. First, a $\pi$ pulse is applied on the second qubit, exchanging $|0\rangle_{B}$ and $|1\rangle_{B}$. This creates only singleparticle phases which can be included into the definition of the single-particle states. If the initial state was $|0\rangle_{A}|0\rangle_{B}$, then after the $\pi$ pulse traps $A_{1}$ and $B_{0}$ would contain no atom. For initial states $|1\rangle_{A}|0\rangle_{B}$ or $|0\rangle_{A}|1\rangle_{B}$, an atom would be either in $A_{1}$ or in $B_{0}$ and as seen before, we could approach and eventually separate $A_{1}$ and $B_{0}$ such that a $n 2 \pi$ pulse is applied with $n$ integer. In this case initial and final state coincide, except for a single-particle phase $\phi_{S}$ which again can be included into the definition of $|1\rangle_{A}$ or $|0\rangle_{B}$. If we started from $|1\rangle_{A}|1\rangle_{B}$, then after the first pulse $A_{1}$ and $B_{0}$ would both be occupied, and during the $n 2 \pi$ pulse the two atoms would collide. For an adiabatic evolution we can neglect not only the probability to populate excited vibrational states, but also to find two atoms in the same trap, since, due to the collisional interaction, these states are not degenerated with states where each atom occupies a different trap [5]. Thus for $a_{t} \neq 0$ initial and final state are the same except for a phase $\phi$ and, in order to realize the desired phase gate operation, we need its collisional part $\phi_{C}=\phi-2 \phi_{S}$ to be an odd multiple of $\pi$. The fidelity of this operation can 
be expressed as $F=\rho\left[\cos \left(\phi_{C}-\pi\right)+1\right] / 2$, which is plotted for the adiabatic regime in Fig. 2(c). Here $\rho$ is the final probability to find the atom in the same state as it was before the $n 2 \pi$ pulse, neglecting the collisional phase. To calculate the collisional phase $\phi_{C}$, we have integrated the two-particle 1D Schrödinger equation replacing $U\left(\vec{r}_{1}, \vec{r}_{2}\right)$ by an effective 1D interaction potential under the assumption that no transverse excitations occur [7]. Finally, to complete the phase gate operation another $\pi$ pulse is applied to the second qubit.

In the case of $2 \mathrm{D}$ arrays of traps, as they are typically realized in the experiment [2], the easiest operation is to move complete columns of microtraps. To realize the gate it is enough to be able to move selectively some columns, with the additional benefit that the operation is applied to many pairs of qubits in parallel which might allow for an easy implementation of error correcting codes. For the side-by-side arrangement, the initial and final $\pi$ pulse can be omitted and only the $2 \pi$ pulse between traps $A_{1}$ and $B_{1}$ is needed. Although conceptually much easier, the implementation in this arrangement demands the ability to move single traps instead of columns, which makes it experimentally more involved.

The SQD scheme allows also to create a maximally entangled state in a straightforward way. Let us consider four traps in the side-by-side arrangement, Fig. 3(a), with the two atoms located in the upper-left and lower-right traps, respectively, i.e., the initial two-particle state is $|0\rangle_{A}|1\rangle_{B}$. During an approach of the traps, Fig. 3(b), the general two-particle state of the system will be

$$
\begin{aligned}
|\Psi(t)\rangle= & \sum_{i, j=0,1} c_{i j}\left|i_{A}\right\rangle\left|j_{B}\right\rangle+\sum_{\alpha=A, B} c_{\alpha}\left|0_{\alpha}\right\rangle\left|1_{\alpha}\right\rangle \\
& +\sum_{i=0,1} \sum_{\alpha=A, B} c_{i \alpha}\left|i_{\alpha}\right\rangle\left|i_{\alpha}\right\rangle .
\end{aligned}
$$

Thus, the state of the system includes the four states of the computational basis, but also double qubit occupation, defined as $\rho_{\mathrm{dq}}=\sum_{\alpha=A, B} c_{\alpha} c_{\alpha}^{*}$, and double trap occupation, $\rho_{\mathrm{dt}}=\sum_{i=0,1} \sum_{\alpha=A, B} c_{i \alpha} c_{i \alpha}^{*}$. In order to create maximally entangled states, the approach of the traps must be both adiabatic and symmetric, i.e., $a(t)=b(t)$. Again, adiabaticity means that the population of excited vibrational states, as well as of double trap occupation states, can be neglected, cf. Fig. 3(c). A symmetric approach results in $\rho_{00}(t)=\rho_{11}(t)=\rho_{\mathrm{dq}}(t) / 2$ during the whole process. In particular, these populations oscillate at the same frequency and, at the oscillation nodes, the state of the system is a combination only of $|0\rangle_{A}|1\rangle_{B}$ and $|1\rangle_{A}|0\rangle_{B}$. Therefore, choosing appropriate parameter values, such as those of Fig. 3, it is possible to obtain a maximally entangled state at the end of the process. Clearly, it is straightforward to generalize this approach to more than two qubits and, consequently, to prepare multiparticle entangled states in one single step.

Although we have assumed harmonic trapping potentials so far, the experimental situation is described
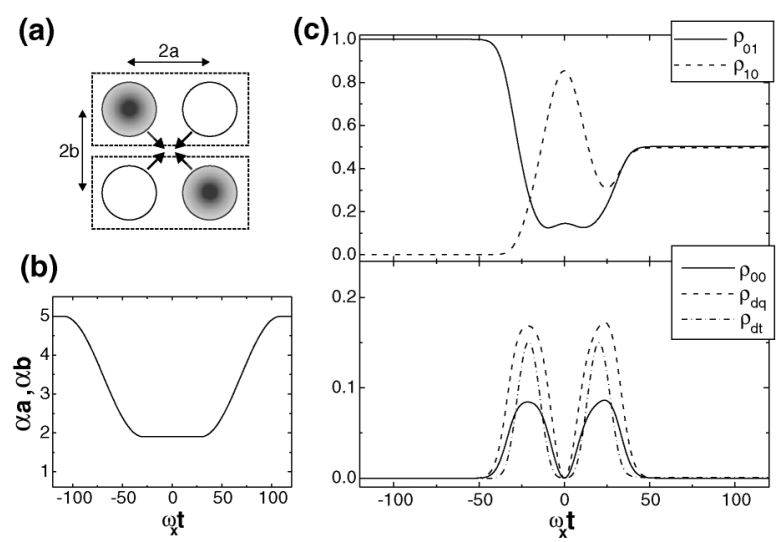

FIG. 3. Creation of a maximally entangled two-qubit state with two ${ }^{87} \mathrm{Rb}$ atoms in a single step: (a) The initial state is $|0\rangle_{A}|1\rangle_{B}$, with $2 a$ and $2 b$ being the separation of the traps in the $x$ and $y$ direction, respectively; (b) we adiabatically and simultaneously, i.e., $a(t)=b(t)$, approach the four traps towards the center of the square; (c) above: population of $|0\rangle_{A}|1\rangle_{B}$ and $|1\rangle_{A}|0\rangle_{B}$; below: population of $|0\rangle_{A}|0\rangle_{B}$ (equal to the population of $|1\rangle_{A}|1\rangle_{B}$ ), double qubit, and double trap occupations. The parameters are: $\alpha a_{\max }=5, \alpha a_{\min }=1.9, \omega_{x} t_{r}=80, \omega_{x} t_{i}=$ 58, $\quad a_{t}=106 a_{0}, \quad \omega_{x}=\omega_{y}=2 \times 10^{5} \mathrm{~s}^{-1}, \quad$ and $\quad \omega_{z}=$ $1.1 \times 10^{6} \mathrm{~s}^{-1}$.

by Gaussian potentials of the form $V(x)=$ $-V_{0} \exp \left[-(1 / 2) m \omega_{x}^{2} x^{2} / V_{0}\right]$. An analysis of the energy eigenvalues and eigenstates of the superposition of two such potentials as a function of the trap separation shows that the cosine function previously used to adiabatically approach the traps leads to values of $t_{r}$ larger by more than 2 orders of magnitude compared to harmonic traps. We have therefore applied the techniques from [8] to optimize the temporal variation of the trap separation while suppressing the population of excited vibrational states. For $V_{0}=200 \hbar \omega_{x}$, Fig. 4(a) shows the result of this optimization for single-qubit operations, where the optimization is done with respect to the symmetric ground and first excited states. We notice that for the minimal distance $2 a_{\min }$ the two traps are no longer separated by a tunneling barrier, but they form a single flat trap. Figure 4(b) shows the population of the right trap, $\rho_{1}$. The error rate due to the excitation of other vibrational states can be made smaller than $1 \%$ for $w_{x} t_{r}>1100$, which is a reduction by 1 order of magnitude compared to the nonoptimized $a(t)$. Further errors could be caused by shaking of the trap positions, the frequency of which in current experiments is 2 or more orders of magnitude below the trapping frequencies, and by intensity fluctuations of the trapping lasers changing the trap depth. In both cases we have found error rates $\lesssim 1 \%$ for amplitudes of a few percent of $a_{\min }$ and $V_{0}$, respectively.

Typical trapping frequencies for state-of-the-art 2D optical microtraps arrays of ${ }^{87} \mathrm{Rb}$ atoms are $10^{5}-10^{6} \mathrm{~s}^{-1}$ in the transverse directions and $10^{4}-10^{5} \mathrm{~s}^{-1}$ along the laser beam direction $[2,6]$. In order not to populate excited vibrational states and neglect double trap 


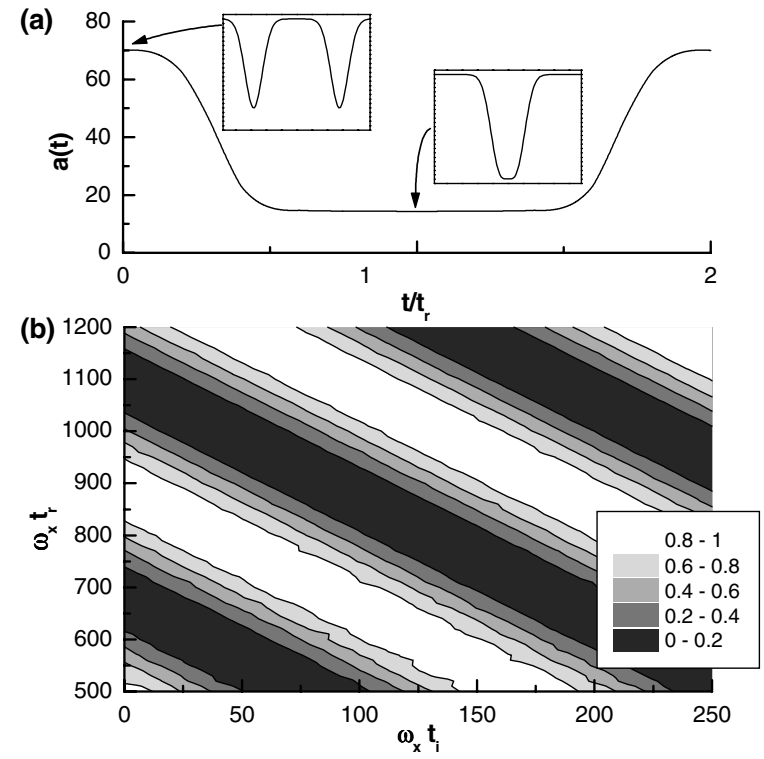

FIG. 4. Single-qubit gates for Gaussian trapping potentials for the following parameters: $\omega_{x}=6 \times 10^{5} \mathrm{~s}^{-1}, \alpha^{-1}=$ $35.4 \mathrm{~nm}, V_{0}=200 \hbar \omega_{x}=0.9 \mathrm{mK} \times \mathrm{k}_{\mathrm{B}}, \alpha a_{\max }=70, \alpha a_{\min }=$ 14.35: (a) The optimized variation of the distance for approaching the traps, insets show the form of the potential; (b) Population $\rho_{1}$ of the right trap showing Rabi-type oscillations.

occupation, the trap displacement has to be adiabatic with respect to the lowest relevant trapping frequency. This adiabaticity condition yields realization times for single and two-qubit operations on the order of $10 \mathrm{~ms}$, respectively. This value should be compared with the typical lifetime of the atoms in the microtraps of $\sim 1 \mathrm{~s}$, and the rate of spontaneous scattering of photons from the trapping laser of $1-10 \mathrm{~s}^{-1}$ [9]. Sideband cooling to a temperature $T \lesssim 1 \mu \mathrm{K}$ with a ground state population of $98.4 \%$ has been achieved in optical lattices with parameters very similar to the ones used here [10], and heating rates below $1 \mu \mathrm{K} / \mathrm{s}$, corresponding to $1 / 80$ and $1 / 16$ vibrational quanta per second in the directions of strong and weak trapping, respectively, have been estimated [11]. Summing up effects of fluctuations of the trap positions, photon scattering, and heating, we can estimate an overall error rate $r \approx 0.02$ for a single-qubit operation with $\omega_{x} t_{r}=1200$, corresponding to a total gate time of $4 \mathrm{~ms}$.

These error rates show that the SDQ configuration with neutral atoms in optical microtraps does not outperform the proposals making use of internal states $[7,12]$ or vibrational states $[5,13]$ taking into account realistic experimental imperfections. But it offers some important practical advantages, which especially lie in the relative simplicity and closeness to experimental implementation: (i) Spontaneous emission leads to decoherence only in a much reduced fashion. As long as the microtraps are moved adiabatically, atoms remain in the internal and external ground state for typical gate times. (ii) There is no momentum transfer in single or two-qubit operations as, in general, it is the case when these operations are realized via laser pulses. This momentum transfer could heat the atoms and, eventually, take them out of the microtrap. (iii) There is no need of state dependent interaction to realize the two-qubit operations. In the SDQ configuration one has that $|\langle 0|\vec{r}| 0\rangle-\langle 1|\vec{r}| 1\rangle| \neq 0$ and, therefore, we can make use of the fact that all interactions are space dependent to realize the gate. (iv) The measurement of the state of the system is straightforward. Optical microtraps can be separated to distances well beyond $\sim 10 \mu \mathrm{m}$, allowing to detect the population by focusing a laser field in one single trap and detecting the fluorescence signal [2]. Finally, (v) single and two-qubit gates are realized by the same kind of operation, i.e., by approaching the microtraps, which implies a strong simplification in the experimental setup.

We note that most of the concepts developed in this Letter can be also applied to quantum dots with the qubit encoded in two tunnel-split ground states and the Coulomb interaction used to realize the qubit operations [14]; and to Josephson junctions based on the charge degree of freedom with the Cooper pairs tunneling coherently through the superconducting junction [15].

This work is supported by the EC through IST projects EQUIP and ACQUIRE, and by the DFG (SPP "Quanteninformationsverarbeitung," SFB 407). We thank D. Bruß, R. Dumke, T. Müther, A. Sanpera, and M. Volk.

[1] P. S. Jessen and I. H. Deutsch, Adv. At. Mol. Opt. Phys. 37, 95 (1996);I. H. Deutsch and P. S. Jessen, Phys. Rev. A 57, 1972 (1998); G. Grynberg and C. Robilliard, Phys. Rep. 355, 335 (2001).

[2] R. Dumke et al., Phys. Rev. Lett. 89, 097903 (2002).

[3] D. Frese et al., Phys. Rev. Lett. 85, 3777 (2000); N. Schlosser et al., Nature (London) 411, 1024 (2001).

[4] M. Greiner et al., Nature (London) 415, 39 (2002).

[5] K. Eckert et al., Phys. Rev. A, 66, 042317 (2002).

[6] G. Birkl et al. Opt. Commun. 191, 67 (2001).

[7] D. Jaksch et al., Phys. Rev. Lett. 82, 1975 (1999); T. Calarco et al., Phys. Rev. A 61, 022304 (2000).

[8] W. Hänsel et al., Phys. Rev. A 64, 063607 (2001).

[9] F. B. J. Buchkremer et al., Phys. Rev. Lett. 85, 3121 (2000).

[10] S. E. Hamann et al., Phys. Rev. Lett. 80, 4149 (1998).

[11] V. Vuletic et al., Phys. Rev. Lett. 81, 5768 (1998).

[12] G. K. Brennen et al., Phys. Rev. Lett. 82, 1060 (1999); D. Jaksch et al., Phys. Rev. Lett. 85, 2208 (2000); I. E. Protsenko et al., Phys. Rev. A 65, 052301 (2002).

[13] E. Charron et al., Phys. Rev. Lett. 88, 077901 (2002).

[14] T. Brandes and T. Vorrath, Phys. Rev. B 66, 075341 (2002); F. Renzoni and T. Brandes, Phys. Rev. B 64, 245301 (2001).

[15] See Y. Makhlin et al., Rev. Mod. Phys. 73, 357 (2001), and references therein. 